\title{
Síntese e caracterização de compósito magnético nanoestruturado contendo óxido de manganês para remoção de íons $\mathrm{Cd}(\mathrm{II})$ do meio aquoso
}

\section{(Synthesis and characterization of a magnetic nanostructured composite containing manganese oxide for removal of Cd (II) from aqueous medium)}

\author{
A. P. Heitmann ${ }^{*}$, G. C. Silva ${ }^{2}$, P. R. P. Paiva ${ }^{2}$, A. M. Ferreira ${ }^{1,2}$ \\ ${ }^{1}$ CEFET-MG-DEMAT, Av. Amazonas 5253, Nova Suiça, Belo Horizonte, MG 30421-169 \\ ${ }^{2}$ Instituto Nacional de Ciência e Tecnologia em Recursos Minerais, Água e Biodiversidade - INCT-CQUA, \\ Av. Antônio Carlos 6627, Pampulha, Belo Horizonte, MG, 31270901 \\ *angelamello@des.cefetmg.br
}

\begin{abstract}
Resumo
$\mathrm{O}$ tratamento de efluentes contendo cádmio é de grande interesse devido à persistência desse contaminante no meio ambiente e às graves doenças que pode causar ao homem. No presente trabalho, foi utilizado o compósito magnético $\mathrm{Mn}_{3} \mathrm{O}_{4} / \mathrm{Fe}_{3} \mathrm{O}_{4}$ como adsorvente do íon cádmio (II) em meio aquoso. O material adsorvente foi sintetizado e caracterizado segundo as técnicas de difração de raios X (DRX), espectroscopia Raman, microscopia eletrônica de transmissão e sorção de $\mathrm{N}_{2}$ (BET). Ênfase foi dada à quantificação das fases do compósito pela análise de DRX combinada ao método Rietveld. O estudo de adsorção foi realizado variando o pH do meio e a concentração do íon cádmio em solução. A análise dos resultados possibilitou verificar que a remoção de $\mathrm{Cd}$ (II) utilizando compósito magnético $\mathrm{Mn}_{3} \mathrm{O}_{4} / \mathrm{Fe}_{3} \mathrm{O}_{4}$ em meio aquoso é dependente do $\mathrm{pH}$ duplicando a medida que o $\mathrm{pH}$ varia de 3 para 7 . O estudo de imobilização do íon contaminante em solução apresentou valores significativos de capacidade máxima de adsorção, de 12,3 $\mathrm{mg}_{\mathrm{Cd}} / \mathrm{g}$ no $\mathrm{pH} 6$ e 13,6 mg $\mathrm{Cd}_{\mathrm{Cd}} / \mathrm{g}$ no $\mathrm{pH} 7$, indicando uma remoção de até $98 \%$ para concentrações baixas do íon, sugerindo que o adsorvente apresenta elevado potencial para remoção de $\mathrm{Cd}(\mathrm{II})$ em meio aquoso.
\end{abstract}

Palavras-chave: adsorção, compósito magnético, cádmio.

\begin{abstract}
The treatment of wastewater containing cadmium is of great interest because of its persistence in the environment and the serious diseases that it can cause to humans. In the present work, the magnetic composite $\mathrm{Mn}_{3} \mathrm{O}_{4} / \mathrm{Fe}_{3} \mathrm{O}_{4}$ was used as adsorbent of cadmium ion in aqueous medium. The adsorbent material was synthesized and characterized according to the XRD, Raman, TEM and $N_{2}$ adsorption (BET) techniques. Emphasis was given to the quantification of the phases of the composite by XRD analysis combined with the Rietveld method. The adsorption study was carried out varying the aqueous medium $p H$ and the concentration of cadmium ions in solution. The results show that the cadmium removal by the $\mathrm{Mn}_{3} \mathrm{O}_{4} / \mathrm{Fe}_{3} \mathrm{O}_{4}$ magnetic composite in aqueous medium is $\mathrm{pH}$ dependent duplicating as the $\mathrm{pH}$ varies from 3 to 7 . The study of immobilization of the contaminant in solution showed significant amounts of adsorption, $12.3 \mathrm{mg}_{\mathrm{Cd}} / \mathrm{g}$ at $\mathrm{pH} 6$ and $13.6 \mathrm{mg}_{\mathrm{Cd}} / \mathrm{g}$ at $\mathrm{pH} 7$ indicating a removal of up to $98 \%$ for low ion concentrations, suggesting that the adsorbent has a high potential for removing Cd(II) in aqueous medium.
\end{abstract}

Keywords: adsorption, magnetic composite, cadmium.

\section{INTRODUÇÃO}

A aplicação de compósitos nanoestruturados nos processos de adsorção visando à remediação ambiental é um campo de interesse crescente, uma vez que a dimensão reduzida desses sistemas sólidos confere a eles uma série de propriedades físico-químicas peculiares que os diferem dos demais materiais. A pequena dimensão das partículas do adsorvente pode oferecer uma elevada área de superfície específica e melhor eficiência de transferência de massa. Combinando essa eficiência de transferência de massa com a utilização do processo de separação magnética, os compósitos nanoestruturados magnéticos possuem elevado potencial para serem aplicadas no sistema de adsorção, sobretudo para grandes volumes de efluentes $[1,2]$.

Adsorventes nanoestruturados baseados em óxidos de manganês têm sido amplamente investigados para adsorção de íons tóxicos como chumbo, cádmio, níquel e zinco do meio aquoso [3, 4]. Esses adsorventes destacam-se por elevada remoção desses íons quando comparada aos outros adsorventes, devido a sua elevada área superficial, estrutura microporosa com tamanho e forma dos poros bem definidos e os grupos funcionais $\mathrm{OH}$ capazes de reagir com os metais e outros íons especificamente. Além disso, o óxido de manganês 
é considerado um material de baixo custo e abundante no meio ambiente. A dimensão nanométrica dificulta a separação do adsorvente da fase aquosa, sendo assim, tornase necessário combiná-lo com outros materiais no intuito de melhorar a separação sólido/líquido e potencializar o efeito adsorvente [5]. Combinando-se as características magnéticas dos óxidos de ferro e adsortivas dos óxidos de manganês nanoestruturados, podem-se obter materiais eficientes e de baixo custo voltados para o tratamento de águas residuais [6]. $\mathrm{O}$ compósito nanoestruturado magnético de $\mathrm{Mn}_{3} \mathrm{O}_{4} / \mathrm{Fe}_{3} \mathrm{O}_{4}$ é uma classe relativamente nova de material que está sendo investigado na remoção de ânions e compostos orgânicos do meio aquoso $[2,7,8]$. Além de possuir elevado potencial de oxidação, o compósito se destaca por se apresentar como nanopartículas com propriedades magnéticas que facilitam a sua separação do meio aquoso após adsorção.

O cádmio está entre os elementos mais tóxicos encontrados na natureza sendo de grande interesse em estudos ambientais. O metal em questão é muito utilizado em processos industriais como na galvanoplastia, na produção de pigmentos, inseticidas e equipamentos eletrônicos, sendo tóxico em baixas concentrações causando sérios problemas de saúde [9]. A intoxicação por esse elemento provoca sintomas específicos, uma vez que o cádmio forma complexos estáveis com os grupos funcionais das enzimas, prejudicando o perfeito funcionamento do organismo, impedindo o transporte de substâncias essenciais ao corpo humano [10]. Com a crescente produção de efluentes é de fundamental importância o estudo de alternativas e o desenvolvimento de novas tecnologias de tratamento que sejam economicamente viáveis e atendam aos limites das legislações existentes [11, 12].

Dentro desse contexto, o objetivo geral do presente trabalho é sintetizar e caracterizar o compósito magnético $\mathrm{Mn}_{3} \mathrm{O}_{4} / \mathrm{Fe}_{3} \mathrm{O}_{4}$ e avaliar seu potencial de adsorção para o íon $\mathrm{Cd}(\mathrm{II})$. O processo de separação magnética sólido-líquido pode viabilizar a aplicação deste compósito em larga escala para tratamento de grandes volumes de águas residuárias. Dentre as diversas técnicas de caracterização utilizadas, ênfase foi dada à difração de raios $\mathrm{X}$ combinada ao refinamento Rietveld que forneceu informações importantes como uma estimativa quantitativa das fases presentes no compósito e a estrutura do adsorvente incluindo os parâmetros de rede do material sintetizado.

\section{MATERIAIS E MÉTODOS}

\section{Preparação do compósito magnético}

$\mathrm{O}$ compósito magnético $\mathrm{Mn}_{3} \mathrm{O}_{4} / \mathrm{Fe}_{3} \mathrm{O}_{4}$ foi sintetizado segundo o procedimento descrito por [2]. Para a preparação desse compósito, $1000 \mathrm{~mL}$ de água deionizada foi aerada por $1 \mathrm{~h}$ e colocada em contato com $45 \mathrm{~mL}$ de uma solução 1 mol $/ \mathrm{L}$ de $\mathrm{MnCl}_{2} \cdot 4 \mathrm{H}_{2} \mathrm{O}$ e $0,6 \mathrm{~g}$ de magnetita $\left(\mathrm{Fe}_{3} \mathrm{O}_{4}\right)$ comercial (Sigma Aldrich $<5 \mu \mathrm{m}$ ). $\mathrm{O} \mathrm{pH}$ da solução foi ajustado para 12 utilizando solução de $0,5 \mathrm{~mol} / \mathrm{L}$ de $\mathrm{KOH}$ em um béquer de $2000 \mathrm{~mL}$, sob agitação mecânica e aeração constantes por
30 min. Após a reação, a solução foi deixada em repouso por 10 min sobre uma placa imantada para garantir a total precipitação do compósito. O compósito foi lavado com água deionizada, seco em temperatura ambiente e macerado utilizando gral e pistilo de ágata.

\section{Caracterização do compósito magnético}

A caracterização do sólido foi feita por meio das técnicas de difração de raios $\mathrm{X}$ (DRX) e refinamento Rietveld, espectroscopia Raman, microscopia eletrônica de transmissão e sorção de nitrogênio para determinação da área superficial pelo método BET. A análise de DRX foi feita no equipamento Shimadzu SSX-550 Superscan. As análises foram conduzidas utilizando anodo de cobre (radiação Cuka, $35 \mathrm{kV} / 40 \mathrm{~mA}$ ), velocidade do goniômetro $0,02^{\circ}$ por passo em $2 \theta$, com tempo de contagem de $5 \mathrm{~s}$ por passo e coletados de $10^{\circ}$ a $90^{\circ}$ em $2 \theta$. A interpretação do difratograma foi efetuada por comparação com padrões do banco de dados PDF02 (ICDD 2003). Para o refinamento Rietveld foi utilizado o programa GSAS e a interface EXPGUI com a função de perfil pseudo-Voigt de ThompsonCox-Hastings e a radiação de fundo ajustada pelo polinômio de Chebyschev. Foram refinados fator de escala, célula unitária, radiação de fundo, assimetria do perfil, parâmetros da largura total à meia-altura a partir dos parâmetros de alargamento instrumental obtidos com um padrão, posição atômica, deslocamentos atômicos isotrópicos e fatores de ocupação dos cátions.

Os espectros Raman foram coletados em equipamento Jobin Yvon Horiba LABRAN-HR800 equipado com um laser de hélio-neônio de $632,8 \mathrm{~nm}, 0,06 \mathrm{~mW}$ de potência, acoplado a um microscópio Olympus BX-41 equipado com lentes de aumento de 10, 50 e 100 vezes. Os espectros foram adquiridos em uma gama de freqüência $100-1100 \mathrm{~cm}^{-1}$ no tempo de aquisição de $60 \mathrm{~s}$, e para aumentar a razão sinal/ ruído, o número de aquisições foi 10.

A medida da área superficial específica do compósito magnético foi realizada pelo método BET através da adsorção de $\mathrm{N}_{2}$, em equipamento Nova 1000 Quantachrome. As amostras foram maceradas e degaseificadas, colocandoas em um tubo de vidro sob vácuo durante $24 \mathrm{~h}$ a $100{ }^{\circ} \mathrm{C}$. Após o resfriamento o tubo foi imerso em nitrogênio líquido, a amostra foi colocada sob vácuo novamente e submetida a valores crescentes de pressão de nitrogênio e, em cada etapa, foram registrados os valores de pressões iniciais e de equilíbrio e a temperatura.

As imagens de microscopia eletrônica de transmissão foram obtidas em equipamento Tecnai-G2-20-FEI 2006. $\mathrm{O}$ espectrômetro com energia dispersiva de raios X (EDS) ThermoNoram (Quest) foi utilizado para detecção elementar.

\section{Avaliação da propriedade de separação magnética}

Os ensaios de turbidez foram realizados com o intuito de verificar a turbidez do material em meio aquoso relacionando-a com a capacidade de separação magnética 
do compósito. Para isso, $1 \mathrm{~g}$ do compósito foi disperso em água, sob agitação constante durante $5 \mathrm{~min}$. Em seguida, uma amostra sedimentou sob influência do campo magnético utilizando o ímã de neodímio e outra amostra sedimentou naturalmente por ação da gravidade. O turbidímetro digital portátil Digimed DM-TU foi utilizado para realizar leituras diretas da turbidez em NTU de ambas as dispersões no intervalo de tempo de 1 a 15 min durante a sedimentação.

\section{Imobilização do cádmio}

Nosexperimentos deadsorção foiavaliado primeiramente, o efeito do $\mathrm{pH}$ na sorção dos íons cádmio. Os ensaios foram realizados com $0,1 \mathrm{~g}$ do compósito magnético em $50 \mathrm{~mL}$ de solução de $\mathrm{Cd}\left(\mathrm{NO}_{3}\right)_{2} \cdot 4 \mathrm{H}_{2} \mathrm{O}$ com concentração de $20 \mathrm{mg} / \mathrm{L}$ e os valores de $\mathrm{pH}$ avaliados no ensaio foram 3, 4, 5, 6 e 7 ajustados com soluções de $0,5 \mathrm{~mol} / \mathrm{L} \mathrm{HCl}$ e $1 \mathrm{~mol} / \mathrm{L} \mathrm{KOH}$. Os ensaios foram conduzidos sob agitação constante de 200 rpm a $25{ }^{\circ} \mathrm{C}$ em um agitador (New Brunswick Scientific Edison, EUA) durante $24 \mathrm{~h}$. Após a reação, os sólidos foram separados das soluções por uma placa imantada. As soluções foram filtradas sob vácuo para garantir que não houvesse partículas suspensas nas amostras para análise química.

Para obtenção das isotermas de adsorção dos íons cádmio, os experimentos de adsorção foram realizados seguindo as condições dos ensaios de $\mathrm{pH}$, em concentrações de 5 a $60 \mathrm{mg} / \mathrm{L}$ na faixa de $\mathrm{pH} 6$ e 7 . As concentrações de cádmio inicial e final foram determinadas por espectroscopia de absorção atômica.

\section{RESULTADOS E DISCUSSÃO}

\section{Caracterização do compósito magnético}

$\mathrm{O}$ difratograma de raios $\mathrm{X}$ obtido para a amostra do compósito $\mathrm{Mn}_{3} \mathrm{O}_{4} / \mathrm{Fe}_{3} \mathrm{O}_{4}$ está representado na Fig. 1, juntamente

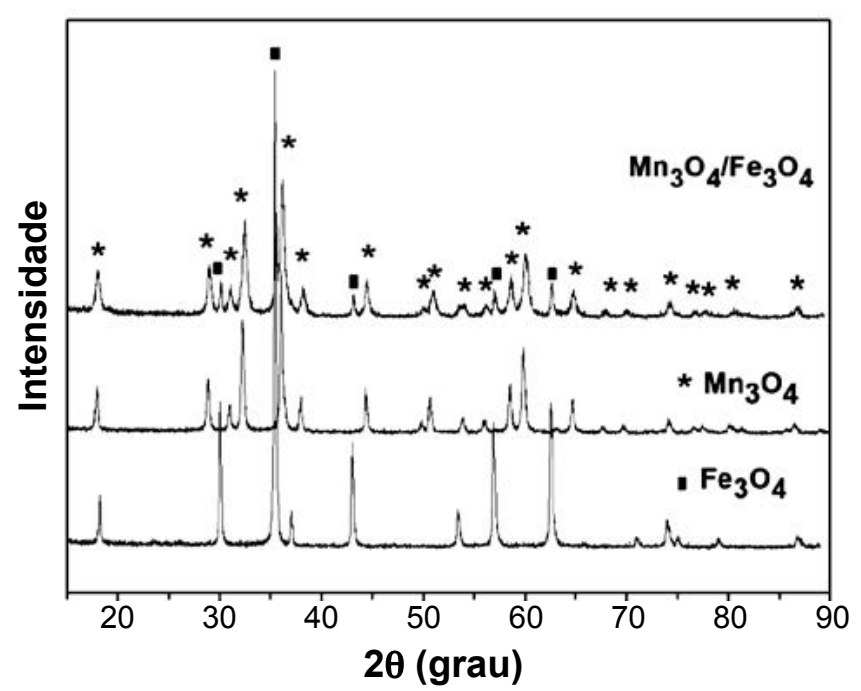

Figura 1: Difratogramas de raios $\mathrm{X}$ do compósito magnético $\mathrm{Mn}_{3} \mathrm{O}_{4} / \mathrm{Fe}_{3} \mathrm{O}_{4}$ e dos padrões $\mathrm{Mn}_{3} \mathrm{O}_{4}$ e $\mathrm{Fe}_{3} \mathrm{O}_{4}$.

[Figure 1: X-ray diffraction patterns of the $\mathrm{Mn}_{3} \mathrm{O}_{4} / \mathrm{Fe}_{3} \mathrm{O}_{4}$ magnetic composite and $\mathrm{Mn}_{3} \mathrm{O}_{4}$ and $\mathrm{Fe}_{3} \mathrm{O}_{4}$ patterns.]

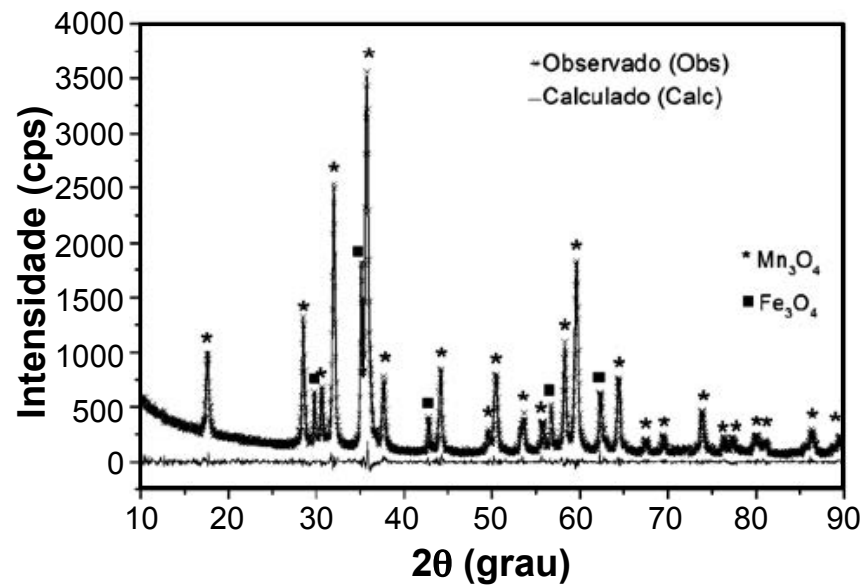

Figura 2: Difratograma refinado pelo método Rietveld do compósito magnético $\mathrm{Mn}_{3} \mathrm{O}_{4} / \mathrm{Fe}_{3} \mathrm{O}_{4}$.

[Figure 2: Rietveld refined diffraction pattern of the $\mathrm{Mn}_{3} \mathrm{O}_{4} / \mathrm{Fe}_{3} \mathrm{O}_{4}$ magnetic composite.]

com os padrões de hausmanita, $\mathrm{Mn}_{3} \mathrm{O}_{4}$ (ICDD 240734) e de magnetita, $\mathrm{Fe}_{3} \mathrm{O}_{4}$ (ICDD 890691) para comparação. A estrutura do compósito magnético foi refinada utilizando o refinamento Rietveld, cujo gráfico está representado na Fig. 2, considerando o grupo espacial da hausmanita I41/amd e o grupo espacial da magnetita $\mathrm{Fd} 3 \mathrm{~m}$. Com o refinamento Rietveld foi possível obter a proporção de $\mathrm{Mn}_{3} \mathrm{O}_{4}(83,69 \%)$ e $\mathrm{Fe}_{3} \mathrm{O}_{4}(16,31 \%)$ no compósito magnético. Além disso, os parâmetros de rede foram determinados sendo $\alpha=\beta=\gamma=90^{\circ}$, $\mathrm{a}=\mathrm{b}=5,764 \AA$ e $\mathrm{c}=9,462 \AA$ para hausmanita $\mathrm{e}, \alpha=\beta=\gamma=90^{\circ}$ e $\mathrm{a}=\mathrm{b}=\mathrm{c}=8,391 \AA$ para magnetita. Os valores calculados são similares aos valores determinados pelo ICDD 240734 para a fase $\mathrm{Mn}_{3} \mathrm{O}_{4}$ e o ICDD 890691 para a fase $\mathrm{Fe}_{3} \mathrm{O}_{4}$. O tamanho médio de cristalito de $\mathrm{Mn}_{3} \mathrm{O}_{4}$ no compósito, obtido pela equação de Scherrer, foi $33 \mathrm{~nm}$. Não é possível estimar o valor médio do cristalito de $\mathrm{Fe}_{3} \mathrm{O}_{4}$ no compósito, uma vez que o material apresenta uma maior distribuição de tamanho $(<5$ $\mu \mathrm{m})$, e a equação de Scherrer não é válida para cristalitos muito grandes [13]. O compósito magnético apresentou

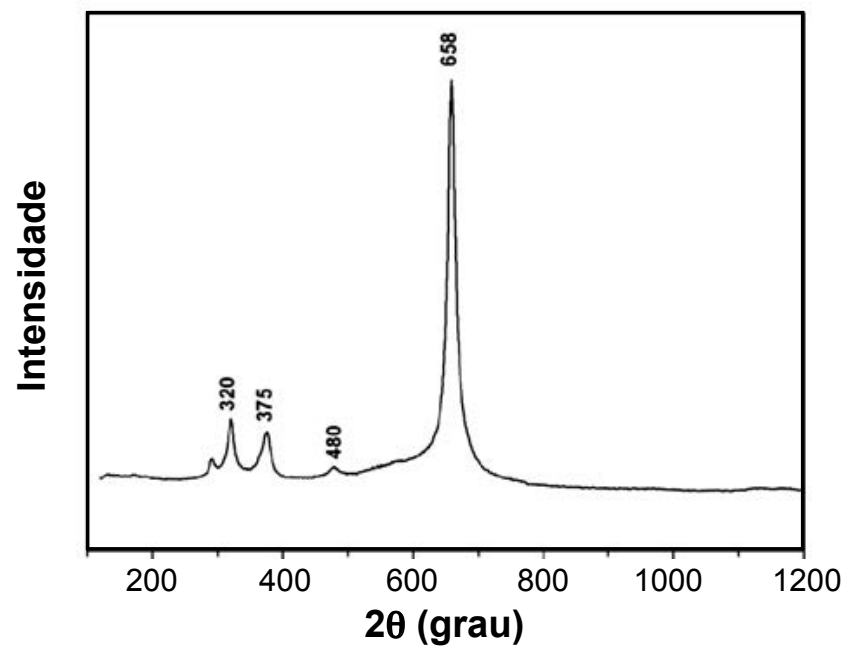

Figura 3: Espectro Raman do compósito magnético $\mathrm{Mn}_{3} \mathrm{O}_{4} / \mathrm{Fe}_{3} \mathrm{O}_{4}$. [Figure 3: Raman spectrum of the $\mathrm{Mn}_{3} \mathrm{O}_{4} / \mathrm{Fe}_{3} \mathrm{O}_{4}$ magnetic composite.] 
elevada cristalinidade $(91,44 \%)$ obtida pelo software XRD Crystallite Size \& Lattice Strain. Os resultados indicam que a síntese na presença da magnetita favorece a formação de uma fase ordenada e única de $\mathrm{Mn}_{3} \mathrm{O}_{4}$.

A área superficial específica obtida para o compósito magnético foi $(44 \pm 9) \mathrm{m}^{2} / \mathrm{g}$, que se encontra mais próximo ao valor estimado do $\mathrm{Mn}_{3} \mathrm{O}_{4}\left(54 \mathrm{~m}^{2} / \mathrm{g}\right)$ quando comparado ao valor estimado para $\mathrm{Fe}_{3} \mathrm{O}_{4}\left(3\right.$ a $\left.6 \mathrm{~m}^{2} / \mathrm{g}\right)[2,14]$. Esse fato pode ser atribuído à presença em maior quantidade de $\mathrm{Mn}_{3} \mathrm{O}_{4}(83,69 \%)$ e a menor quantidade de $\mathrm{Fe}_{3} \mathrm{O}_{4}(16,31 \%)$ no compósito magnético e sugere o recobrimento das partículas de $\mathrm{Fe}_{3} \mathrm{O}_{4}$ pelas partículas de $\mathrm{Mn}_{3} \mathrm{O}_{4}$.

$\mathrm{O}$ espectro Raman obtido para o compósito magnético $\mathrm{Mn}_{3} \mathrm{O}_{4} / \mathrm{Fe}_{3} \mathrm{O}_{4}$ está representado na Fig. 3, na qual se observam três bandas localizadas em 658,375 e $320 \mathrm{~cm}^{-1}$, que estão de acordo com a literatura de referência para o espectro Raman de $\mathrm{Mn}_{3} \mathrm{O}_{4}$ [15]. O pico mais fino e de maior intensidade em $658 \mathrm{~cm}^{-1}$ é característico da espécie $\mathrm{Mn}_{3} \mathrm{O}_{4}$ [16]. Essa banda relaciona-se à vibração $\mathrm{Mn}-\mathrm{O}$ dos íons divalentes do manganês em coordenação tetraédrica, sendo típico de uma estrutura em espinélio [7]. As bandas menores que estão localizadas em 320 e $375 \mathrm{~cm}^{-1}$ correspondem a vibração de deformação de $\mathrm{Mn}_{3} \mathrm{O}_{4}$ e o sinal fraco em $480 \mathrm{~cm}^{-1}$ é referente ao estiramento assimétrico Mn-O-Mn atribuída a essa estrutura. Não é possível identificar no compósito as bandas de $\mathrm{Fe}_{3} \mathrm{O}_{4}$ em torno de $660-680 \mathrm{~cm}^{-1}, 550-570 \mathrm{~cm}^{-1} \mathrm{e}$ $300-320 \mathrm{~cm}^{-1}$ [17-19]. Essas bandas não são observadas, pois o Raman é uma técnica de superfície e, no compósito, as partículas de $\mathrm{Fe}_{3} \mathrm{O}_{4}$ são revestidas pelas partículas de $\mathrm{Mn}_{3} \mathrm{O}_{4}$.

A Fig. 4 mostra partículas de $\mathrm{Mn}_{3} \mathrm{O}_{4}$ presentes no compósito. As partículas de $\mathrm{Fe}_{3} \mathrm{O}_{4}$ não foram identificadas

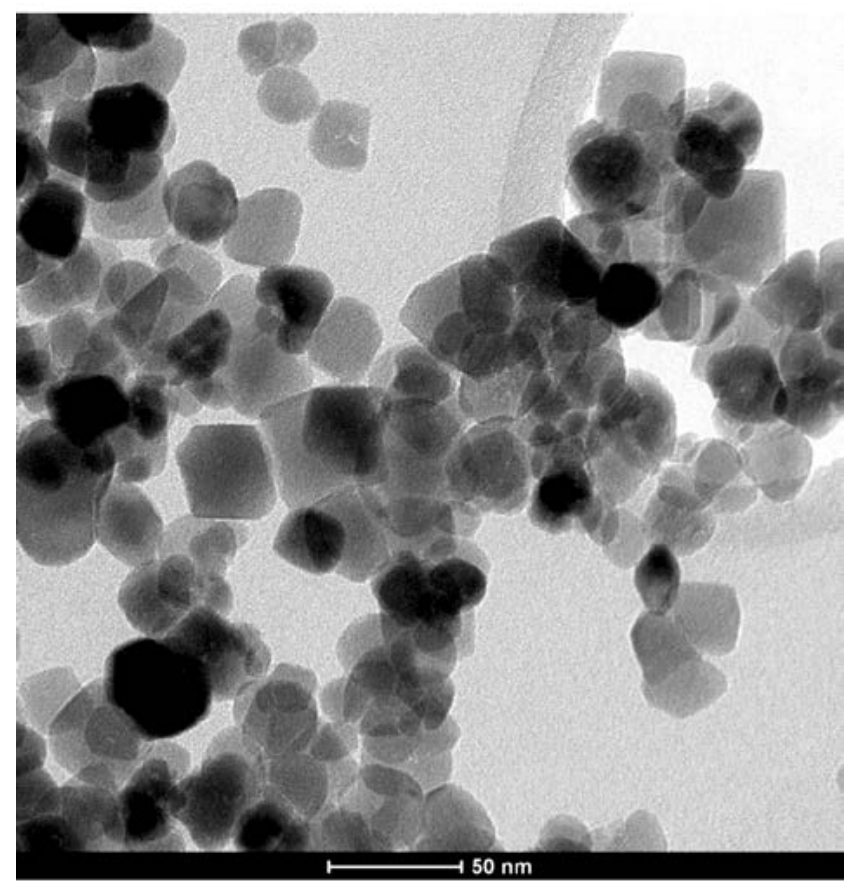

Figura 4: Imagem MET das partículas de $\mathrm{Mn}_{3} \mathrm{O}_{4}$ no compósito magnético $\mathrm{Mn}_{3} \mathrm{O}_{4} / \mathrm{Fe}_{3} \mathrm{O}_{4}$.

[Figure 4: Transmission electron microscopy image of the $\mathrm{Mn}_{3} \mathrm{O}_{4}$ particles in the magnetic composite $\mathrm{Mn}_{3} \mathrm{O}_{4} \mathrm{Fe}_{3} \mathrm{O}_{4}$.] nessa imagem de microscopia eletrônica de transmissão, provavelmente devido à forma como foi realizada a preparação da amostra. Na preparação, foi produzida uma suspensão com o compósito que foi gotejada sobre um substrato. Devido à diferença de densidade entre os seus minerais constituintes, $\mathrm{Fe}_{3} \mathrm{O}_{4}\left(\mathrm{~d}=5,15 \mathrm{~g} / \mathrm{cm}^{3}\right)$ e $\mathrm{Mn}_{3} \mathrm{O}_{4}$ $\left(\mathrm{d}=4,84 \mathrm{~g} / \mathrm{cm}^{3}\right)$ é provável que apenas as partículas menos densas foram amostradas e analisadas no MET. Pode-se observar um agregado de nanopartículas de $\mathrm{Mn}_{3} \mathrm{O}_{4}$ sem orientação específica e com a predominância da morfologia octaédrica. Estima-se o tamanho das nanopartículas em torno de 25-30 nm (Fig. 4) próximo ao valor de $33 \mathrm{~nm}$ calculado pelo difratograma de raios $\mathrm{X}$ para a fase $\mathrm{Mn}_{3} \mathrm{O}_{4}$ no compósito magnético.

Com o intuito de verificar a morfologia de $\mathrm{Fe}_{3} \mathrm{O}_{4}$ no compósito, outras preparações foram preparadas para análise no MET. No canto direito da Fig. 5a (destacado por uma seta), pode-se observar um cristal de morfologia octaédrica com tamanho superior, em torno de $100 \mathrm{~nm}$. A análise de EDS desse cristal, apresentada na Fig. 5b, confirma a presença de ferro e manganês, mostrando que a partícula maior de $\mathrm{Fe}_{3} \mathrm{O}_{4}$ é revestida por partículas menores de $\mathrm{Mn}_{3} \mathrm{O}_{4}$.

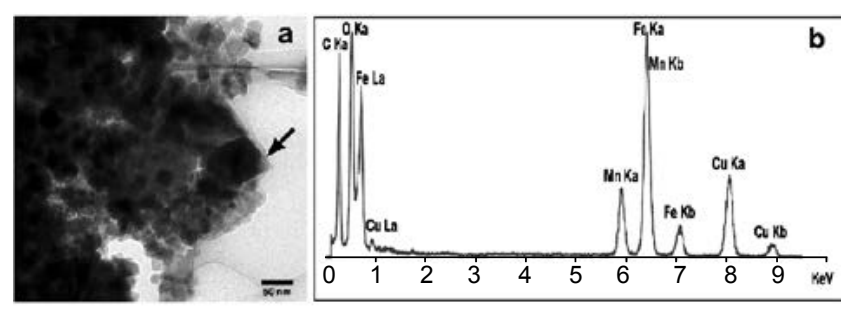

Figura 5: (a) Imagem MET do compósito magnético e (b) análise de EDS da partícula destacada em (a).

[Figure 5: (a)Transmission electron microscopy image of the $\mathrm{Mn}_{3} \mathrm{O}_{4} / \mathrm{Fe}_{3} \mathrm{O}_{4}$ magnetic composite (b) EDS analysis of the particle highlighted in (a).]

\section{Avaliação da propriedade de separação magnética}

Na Tabela I são apresentados os valores de turbidez do compósito magnético disperso em água submetido à sedimentação natural e à sedimentação magnética em um intervalo de tempo de $15 \mathrm{~min}$.

Na Fig. 6 estão representadas as imagens do ensaio de turbidez nos tempos de 1 a 15 min para ambas as condições de sedimentação.

Com os resultados apresentados acima, pode-se observar que a sedimentação natural ocorre mais lentamente do que a sedimentação magnética, uma vez que durante o intervalo de 15 min a primeira apresenta valores de turbidez que são aproximadamente o dobro da segunda. Esse resultado pode ser confirmado pelas imagens apresentadas na Fig. 6, onde para 1 min já é possível observar uma diferença significativa de turbidez entre as amostras. Portanto, pode-se afirmar que o compósito magnético quando disperso em água se apresenta na forma de partículas muito finas, dificultando o processo de sedimentação natural. Dessa forma, a sedimentação magnética tornou-se uma alternativa promissora, uma vez 
Tabela I - Valores da turbidez do compósito magnético disperso em água.

[Table I - Turbidity values of the dispersed magnetic composite in water.]

\begin{tabular}{ccc}
\hline Tempo (min) & $\begin{array}{c}\text { Turbidez (NTU) } \\
\text { Sedimentação } \\
\text { Natural }\end{array}$ & $\begin{array}{c}\text { Turbidez (NTU) } \\
\text { Sedimentação } \\
\text { Magnética }\end{array}$ \\
\hline 1 & 29,1 & 11,4 \\
5 & 22,4 & 8,3 \\
10 & 16,2 & 6,5 \\
15 & 12,4 & 5,8 \\
\hline
\end{tabular}

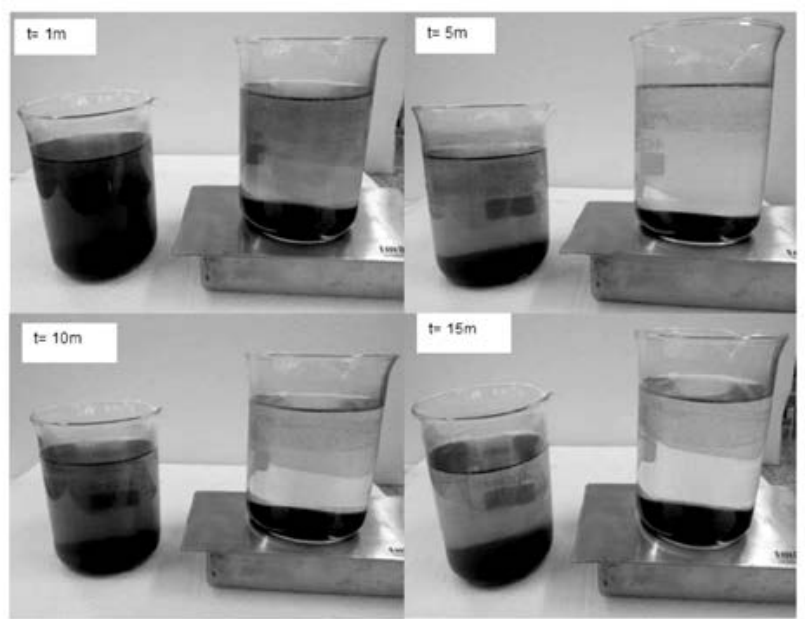

Figura 6: Imagens do ensaio de turbidez para avaliação da separação magnética.

[Figure 6: Turbidity test images to evaluate the magnetic separation.]

que apresentou maior eficiência na separação das partículas do compósito do meio aquoso.

\section{Imobilização do cádmio}

A influência do valor do $\mathrm{pH}$ na capacidade de sorção do cádmio em meio aquoso pode ser verificado na Fig. $7 . \mathrm{O}$ efeito do $\mathrm{pH}$ da solução contendo o íon metálico tem sido considerado uma variável importante que afeta mais significativamente a adsorção do metal no material adsorvente. Na Fig. 7 é mostrada a eficiência de remoção do $\mathrm{Cd}(\mathrm{II})$ em relação ao valor do $\mathrm{pH}$ da solução de equilíbrio na faixa de 3-7, revelando que a adsorção do cádmio no compósito $\mathrm{Mn}_{3} \mathrm{O}_{4} / \mathrm{Fe}_{3} \mathrm{O}_{4}$ é influenciada pelas condições de $\mathrm{pH}$ do meio, cuja remoção do íon com a variação do $\mathrm{pH} 3$ para 7 foi de $3,2 \mathrm{mg} / \mathrm{g}$ para $7,0 \mathrm{mg} / \mathrm{g}$, ou seja, de $35 \%$ para $70 \%$. A maior capacidade de sorção dos íons cádmio ocorreu no valor de $\mathrm{pH}$ em torno de 7 , pois em baixos valores de $\mathrm{pH}$, a remoção de íons $\mathrm{Cd}$ (II) foi inibida possivelmente como resultado da competição entre os íons $\mathrm{H}^{+}$e $\mathrm{Cd}(\mathrm{II})$ sobre os sítios ativos, com uma predominância de íons $\mathrm{H}^{+}$. Com o aumento do $\mathrm{pH}$, a densidade de carga negativa sobre a superfície do compósito aumenta devido a desprotonação da superfície, e assim, a adsorção dos íons Cd(II) é elevada [20].

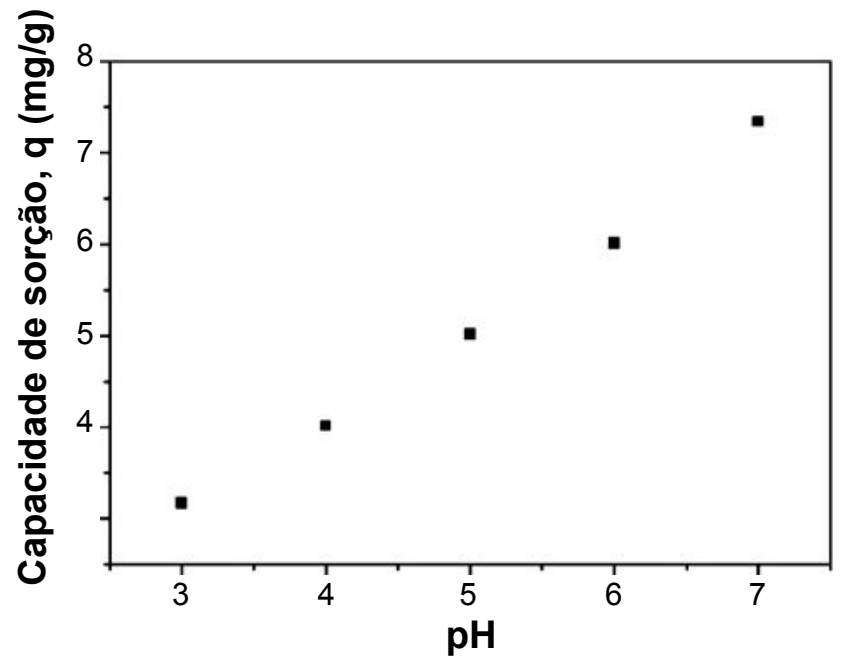

Figura 7: Efeito do pH na adsorção de $\mathrm{Cd}(\mathrm{II})$ em meio aquoso. [Figure 7: pH effect on the Cd(II) adsorption in aqueous medium.]

O estudo de adsorção do Cd(II) foi realizado com o intuito de avaliar o efeito da concentração do contaminante no meio aquoso e a capacidade de remoção desse íon utilizando o compósito magnético. Para tal, foram determinadas isotermas de adsorção em pH 6 e 7, ajustadas ao modelo de Langmuir-Freundlich (eq. A) [21].

$$
\mathrm{q}=\mathrm{b} \cdot \mathrm{Q}_{\mathrm{m}} \cdot \mathrm{C}_{\mathrm{e}}{ }^{1 / \mathrm{n}} /\left[1+\mathrm{b} \cdot \mathrm{C}_{\mathrm{e}}{ }^{1 / \mathrm{n}}\right]
$$

na qual q é a quantidade adsorvida do adsorvato no equilíbrio $(\mathrm{mg} / \mathrm{g}), \mathrm{C}_{\mathrm{e}}$ a concentração do adsorvato no equilíbrio (mg/L), b a constante de adsorção de LangmuirFreundlich (L/mg), $\mathrm{Q}_{\mathrm{m}}$ a capacidade máxima de adsorção $(\mathrm{mg} / \mathrm{g})$ e $1 / \mathrm{n}$ o parâmetro de heterogeneidade. A capacidade máxima de adsorção $\left(\mathrm{Q}_{\mathrm{m}}\right)$ e a afinidade dos íons cádmio com o adsorvente foram obtidas do ajuste dos dados. $\mathrm{O} \chi^{2}$ (goodness-of-fit) é um parâmetro estatístico utilizado para avaliar a qualidade do ajuste. $\mathrm{O}$ valor desse parâmetro deve ser o menor possível (menor que $1 \%$, ou seja, 0,01 ). Os

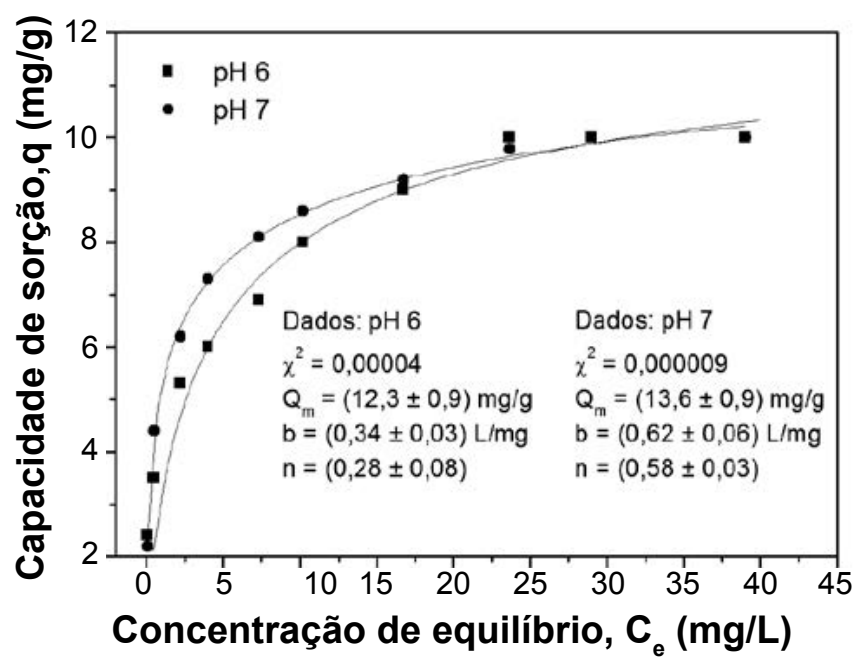

Figura 8: Isotermas de adsorção para o Cd(II) em solução aquosa. [Figure 8: Adsorption isotherms of Cd(II) in aqueous solution.] 
valores de $\chi^{2}$ obtidos no presente trabalho indicam (Fig. 8) uma boa qualidade do ajuste. Em relação à isoterma de Langmuir-Freundlich, o parâmetro $b$ representa afinidade do íon pelos sítios do material adsorvente e a energia livre no processo de adsorção [22]. O valor de $b$ obtido para a adsorção de $\mathrm{Cd}(\mathrm{II})$ no compósito é de $0,34 \mathrm{~L} / \mathrm{mg}$ quando ocorre em $\mathrm{pH} 6$ e $0,62 \mathrm{~L} / \mathrm{mg}$ para o $\mathrm{pH} 7$, mostrando que apesar da capacidade de adsorção do íon cádmio, no pH 6, apresentar valor próximo a adsorção no $\mathrm{pH} 7$, o íon cádmio apresenta maior afinidade pelo compósito quando a adsorção ocorre no $\mathrm{pH} 7$, apresentando uma maior energia de ligação entre as espécies.

A capacidade máxima de sorção do íon metálico, $\mathrm{Q}_{\mathrm{m}}$, obtida pela isoterma de Langmuir-Freundlich apresentou valor $12,3 \mathrm{mg}_{\mathrm{Cd}} / \mathrm{g}\left(0,11 \mathrm{mmol}_{\mathrm{Cd}} / \mathrm{g}\right)$ para adsorção no $\mathrm{pH} 6$ e 13,6 mg $\mathrm{Cd}_{\mathrm{Cd}} / \mathrm{g}\left(0,12 \mathrm{mmol}_{\mathrm{Cd}} / \mathrm{g}\right)$ para adsorção no $\mathrm{pH} 7$, não havendo evidências de precipitação (Fig. 8). Os valores obtidos são muito próximos em ambos os valores de $\mathrm{pH}$. Comparando esses valores com valores encontrados na literatura para adsorção de $\mathrm{Cd}(\mathrm{II})$, observamos que são maiores do que a capacidade máxima de adsorção do metal em hausmanita no $\mathrm{pH} 6,3\left(4,34 \mathrm{mg}_{\mathrm{Cd}} / \mathrm{g}\right)$ [23], em goethita e boemita sintética em pH $6\left(5,18 \mathrm{mg}_{\mathrm{Cd}} / \mathrm{g}\right.$ e $3,55 \mathrm{mg}_{\mathrm{Cd}} / \mathrm{g}$, respectivamente) [24] e em nanopartículas de magnetitamaghemita na faixa de $\mathrm{pH} 7-8\left(2,70 \mathrm{mg}_{\mathrm{Cd}} / \mathrm{g}\right)$ [25].

Em um trabalho prévio, o compósito magnético $\mathrm{Mn}_{3} \mathrm{O}_{4} /$ $\mathrm{Fe}_{3} \mathrm{O}_{4}$ foi sintetizado e aplicado para imobilização da espécie As(III) em meio aquoso obtendo uma capacidade de adsorção de $14 \mathrm{mg}_{\mathrm{As}} / \mathrm{g}\left(0,0048 \mathrm{mmol}_{\mathrm{As}} / \mathrm{g}\right) \mathrm{em} \mathrm{pH} 5$, apresentando elevada afinidade pelo material que é desejado para remoção de concentrações traços de contaminantes [2]. Além disso, observou-se que a hausmanita $\left(\mathrm{Mn}_{3} \mathrm{O}_{4}\right)$ favorece a oxidação do $\mathrm{As}(\mathrm{III})$ para o $\mathrm{As}(\mathrm{V}) \mathrm{e}$, conseqüentemente, $\mathrm{Mn}(\mathrm{III})$ é reduzido a $\mathrm{Mn}(\mathrm{II})$ e a espécie $\mathrm{As}(\mathrm{V})$ é adsorvida no material. Entretanto, a adsorção do $\mathrm{Cd}(\mathrm{II})$ não afeta o estado de oxidação do íon Mn nos minerais de manganês, sendo assim é improvável que o cádmio esteja sofrendo oxidação ou redução durante o processo [26]. A adsorção do cádmio no compósito magnético ocorre acima do ponto de carga zero $(6,2)$, ou seja, a superfície do adsorvente se encontra desprotonada favorecendo a atração eletrostática do íon Cd(II) no material nos sítios livres para adsorção, embora não se pode afirmar que este seja o mecanismo predominante de adsorção do íon no adsorvente.

A porcentagem de remoção do íon cádmio no compósito magnético em meio aquoso está representada na Fig. 9, onde se observa que a remoção de Cd(II) em compósito magnético é elevada para concentrações baixas do íon em solução. As concentrações de até $20 \mathrm{mg} / \mathrm{L}$ apresentam uma taxa de remoção bastante significativa em torno de $80 \%$ para ambos os valores de $\mathrm{pH}$. Em concentração de aproximadamente $5 \mathrm{mg} / \mathrm{L}$ de $\mathrm{Cd}(\mathrm{II})$ a remoção do íon atinge o máximo de $98 \%$ em pH 7 e $90 \%$ em pH 6 e a medida que aumenta a concentração do íon Cd(II) em solução essa porcentagem diminui para em torno de $30 \%$ no $\mathrm{pH} 6$ e 7 . Esse comportamento é esperado, uma vez que em concentrações mais baixas do íon, o compósito magnético apresenta sítios

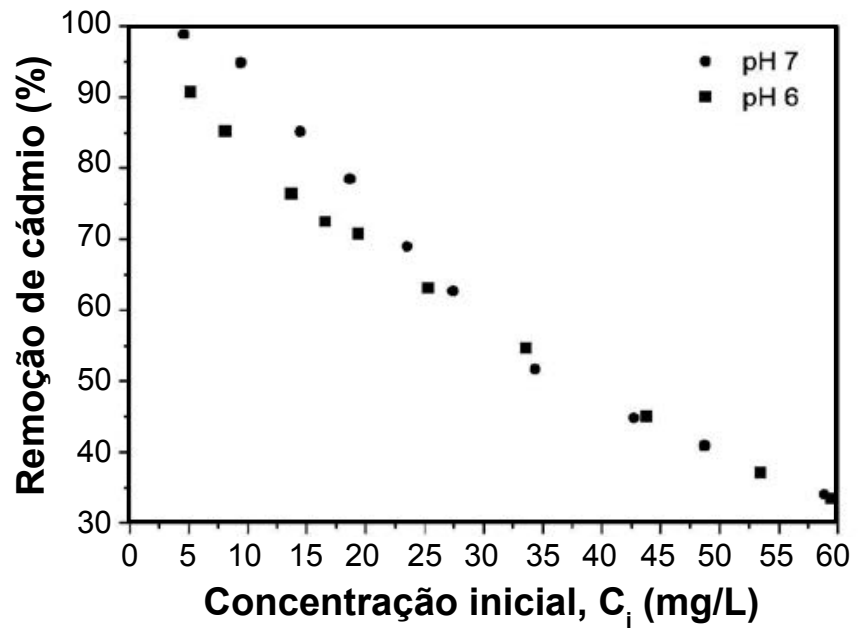

Figura 9: Remoção de Cd(II) pelo compósito magnético em meio aquoso.

[Figure 9: Cd(II) removal by the magnetic composite in aqueous medium.]

ativos livres para adsorção e quando essa concentração aumenta os sítios ativos já se encontram ocupados, em sua maioria, ocasionando uma menor eficiência de remoção. Dessa maneira, sugere-se que o compósito magnético pode ser considerado um adsorvente eficiente para remoção de concentrações traços do contaminante no meio aquoso tornando-o bastante atrativo para ser aplicado em processos de adsorção em escala industrial.

\section{CONCLUSÃO}

O compósito magnético foi sintetizado e a sua caracterização mostrou que há predominância da hausmanita $\left(\mathrm{Mn}_{3} \mathrm{O}_{4}\right)$ em relação à magnetita $\left(\mathrm{Fe}_{3} \mathrm{O}_{4}\right)$ e que ocorre o recobrimento dessas partículas de $\mathrm{Fe}_{3} \mathrm{O}_{4}$ pelas partículas de $\mathrm{Mn}_{3} \mathrm{O}_{4}$. A difração de raios $\mathrm{X}$ associada ao Rietveld mostrou ser uma ferramenta de caracterização muito importante na quantificação das fases, bem como na determinação dos parâmetros de rede dos componentes do compósito, confirmando ser a hausmanita o único óxido de manganês formado. A adsorção do cádmio no compósito magnético revela que a capacidade de adsorção do íon aumenta à medida que se eleva o valor do $\mathrm{pH}$ de 3 para 7 . As isotermas de adsorção do $\mathrm{Cd}(\mathrm{II})$ foram obtidas em $\mathrm{pH} 6$ e 7, sendo melhor ajustadas pelo modelo Langmuir-Freundlich. A capacidade máxima de adsorção foi similar para $\mathrm{pH} 6 \mathrm{e}$ $\mathrm{pH} 7$, sendo os valores correspondentes a $\mathrm{Q}_{\mathrm{m}}=12,3 \mathrm{mg} / \mathrm{g}$ e $\mathrm{Q}_{\mathrm{m}}=13,6 \mathrm{mg} / \mathrm{g}$, respectivamente. Em concentrações baixas, a remoção foi mais eficiente no $\mathrm{pH} 7$, chegando a $98 \%$ de remoção de $\mathrm{Cd}$ (II) em solução indicando que o compósito magnético apresenta potencial para ser utilizado na remoção de concentrações traços em meio aquoso. Os ensaios de turbidez mostraram que a sedimentação magnética tornouse uma alternativa promissora, uma vez que apresentou maior eficiência na separação das partículas do compósito do meio aquoso. Conclui-se que o compósito magnético $\mathrm{Mn}_{3} \mathrm{O}_{4} / \mathrm{Fe}_{3} \mathrm{O}_{4}$ é promissor para aplicação em larga escala 
para tratamento de águas residuais devido à facilidade de sua separação do meio aquoso pela simples aplicação de um campo magnético, dispensando assim, a difícil etapa de separação por filtração de partículas nanoestruturadas.

\section{AGRADECIMENTOS}

Ao CNPq e CAPES pelo suporte financeiro, ao Departamento de Engenharia de Materiais do CEFET MG, ao Departamento de Engenharia Metalúrgica e de Materiais da UFMG e ao INCT-Acqua pela infraestrutura disponibilizada para realização deste trabalho.

\section{REFERÊNCIAS}

[1] C. F. Chang, P. H. Lin, W. Höll, "Aluminum-type superparamagnetic adsorbents: synthesis and application on fluoride removal", Colloids Surface 280 (2006) 194.

[2] G. C. Silva, F. S. Almeida, A. M. Ferreira, V. S. T. Ciminelli, "Preparation and application of a magnetic composite $\left(\mathrm{Mn}_{3} \mathrm{O}_{4} / \mathrm{Fe}_{3} \mathrm{O}_{4}\right)$ for removal of $\mathrm{As}(\mathrm{III})$ from aqueous solutions”, Mater. Res. 15 (2012) 403.

[3] Q. Qin, Q. Wang, D. Fu, J. Ma, “An efficient approach for $\mathrm{Pb}(\mathrm{II})$ and $\mathrm{Cd}(\mathrm{II})$ removal using manganese dioxide formed in situ", Chem. Eng. J. 172 (2011) 68.

[4] Q. Su, B. Pan, S. Wan, W. Zhang, L. Lv, "Use of hydrous manganese dioxide as a potential sorbent for selective removal of lead, cadmium and zinc ions from water", J. Colloid Interface Sci. 349 (2010) 607.

[5] M. Hua, S. Zhang, B. Pan, W. Zhang, L. Lv, Q. Zhang, "Heavy metal removal from water/wastewater by nanosized metal oxides: a review", J. Hazardous Mater. 211 (2012) 317.

[6] H. Chen, J. He, "Facile synthesis of monodisperse manganese oxide nanostructures and their application in water treatment", J. Phys. Chem. C 112 (2008) 17540.

[7] G. C. Silva, F. S. Almeida, A. M. Ferreira, V. S. T. Ciminelli, M. S. S. Dantas, "Raman and IR spectroscopic investigation of As adsorbed on $\mathrm{Mn}_{3} \mathrm{O}_{4}$ magnetic composites", Spectrochim. Acta Part A: Molecular Biomolecular Spectroscopy 100 (2013) 161.

[8] G. C. Silva, V. S. T. Ciminelli, A. M. Ferreira, N. C. Pissolati, P. R. P. Paiva, J. L. López, "A facile synthesis of $\mathrm{Mn}_{3} \mathrm{O}_{4} / \mathrm{Fe}_{3} \mathrm{O}_{4}$ superparamagnetic nanocomposites by chemical precipitation: Characterization and application in dye degradation", Mater. Res. Bull. 49 (2014) 544.

[9] L. M. M. Colla, M. Hekemeir, A. S. L. Gil, "Biossorção de cádmio e produção de biossurfactantes por fungos filamentosos em fermentação submersa", Rev. CIATEC 4 (2012) 1.

[10] M. R. M. P Aguiar, A. C. Novaes, A. W. S. Guarino, "Remoção de metais pesados de efluentes industriais por aluminossilicatos", Química Nova 25 (2002) 1145.

[11] C. Quintelas, B. Fernandes, J. Castro, H. Figueiredo, T. Tavares, "Biosorption of $\mathrm{Cr}(\mathrm{VI})$ by three different bacterial species supported on granular activated carbon - A comparative study", J. Hazardous Mater. 153 (2008) 799.

[12] T. K. Naya, A. K. Bhattacharya, S. K. Das, "Adsorption of $\mathrm{Cd}(\mathrm{II})$ and $\mathrm{Pb}$ (II) from aqueous solutions on activated alumina", J. Colloid and Interface Sci. 33 (2009) 14.

[13] R. E. Dinnebier, S. J. L. Billingue, Powder diffraction theory and practice, The Royal Society of chemistry, $1^{\text {st }}$ Ed., The Royal Society Chemistry, London, UK (2008) 604.

[14] C. A. C. Rosas, M. Franzreb, F. Valenzuela, W. H. Höll, "Magnetic manganese dioxide as an amphoteric adsorbent for removal of harmful inorganic contaminants from water", Reactive Functional Polymers 70 (2010) 516.

[15] Y. Liu, Z. Liu, G. Wang, "Preparation of $\mathrm{Mn}_{3} \mathrm{O}_{4}$ nanowires by calcining the precursor powders synthesized in a novel inverse microemulsion", Appl. Phys. A 76 (2003) 1117.

[16] L. X. Yang, Y. J. Zhu, H. Tong, W. W. Wang, G. F. Cheng, "Low temperature synthesis of $\mathrm{Mn}_{3} \mathrm{O}_{4}$ polyhedral nanocrystals and magnetic study", J. Solid State Chem. 179 (2006) 1225.

[17] Y. F. Han, F. Chen, Z. Y. Zhong, K. Ramesh, E. Widjaja, L. W. Chen, "Synthesis and characterization of $\mathrm{Mn}_{3} \mathrm{O}_{4}$ and $\mathrm{Mn}_{2} \mathrm{O}_{3}$ nanocrystals on SBA-15: Novel combustion catalysts at low reaction temperatures", Catalysis Comm. 7 (2006) 739.

[18] C. M. Julien, M. Massot, C. Poinsignon, "Lattice vibrations of manganese oxides: part I periodic structures", Spectrochim. Acta - Part A 60 (2004) 689.

[19] O. N. Shebanova, P. Lazor, "Raman study of magnetite $\left(\mathrm{Fe}_{3} \mathrm{O}_{4}\right)$ : laser-induced thermal effects and oxidation", J. Raman Spectroscopy 34 (2003) 845.

[20] S. R. Taffarel, J. Rubio, "On the removal of $\mathrm{Mn}^{2+}$ ions by adsorption onto natural and activated Chilean zeolites", Minerals Eng. 22 (2009) 336.

[21] A. Debrassi, M. C. T. Largura, C. A. Rodrigues, "Adsorção do corante vermelho congo por derivados da o-carboximetilquitosana hidrofobicamente modificados", Quim. Nova 34 (2011) 764.

[22] L. Dong, Z. Zhu, H. Ma, Y. Qiu, J. Zhao, "Simultaneous adsorption of lead and cadmium on $\mathrm{MnO}_{2}$-loaded resin", J. Environ. Sci. 22 (2010) 225.

[23] C. A. Backes, R. G. Mclaren, A. W. Rate, R. S. Swift, "Kinetics of cadmium and cobalt desorption from iron and manganese oxides", Soil Sci. Soc. Am. J. 59 (1995) 778.

[24] F. G. Correa, N. G. C. Capulin, M. T. Olguín, C. E. A. León, "Comparison of the $\mathrm{Cd}(\mathrm{II})$ adsorption processes between boehmite $(\alpha-\mathrm{AlOOH})$ and goethite $(\alpha-\mathrm{FeOOH})$ ", Chem. Eng. J. 171 (2011) 1027.

[25] S. R. Chowdhury, E. K. Yanful, "Kinetics of Cd(II) uptake by mixed maghemite-magnetite nanoparticles", J. Environ. Manag. 129 (2013) 642.

[26] L. Bochatay, "Metal ion coordination at the watermanganite $(\gamma-\mathrm{MnOOH})$ interface I. An EXAFS study of cadmium (II)", J. Colloid Interface Sci. 229 (2000) 584.

(Rec. 08/01/2014, Rev. 19/03/2014, 17/04/2014, Ac. $17 / 06 / 2014)$ 